ARTICLE

Received 17 Mar 2015 | Accepted 17 Aug 2015 | Published 23 Sep 2015

DOI: $10.1038 /$ ncomms 9387

OPEN

\title{
Direct quantitative detection of Doc2b-induced hemifusion in optically trapped membranes
}

Ineke Brouwer ${ }^{1, \star}$, Asiya Giniatullina ${ }^{2, \star}$, Niels Laurens ${ }^{1}$, Jan R.T van Weering ${ }^{2}$, Dirk Bald ${ }^{3}$, Gijs J.L. Wuite ${ }^{1, \dagger}$ $\&$ Alexander J. Groffen ${ }^{2, \dagger}$

$\mathrm{Ca}^{2+}$-sensor proteins control the secretion of many neuroendocrine substances. Calcium-secretion coupling may involve several mechanisms. First, $\mathrm{Ca}^{2+}$-dependent association of their tandem C2 domains with phosphatidylserine may induce membrane curvature and thereby enhance fusion. Second, their association with SNARE complexes may inhibit membrane fusion in the absence of a $\mathrm{Ca}^{2}+$ trigger. Here we present a method using two optically trapped beads coated with SNARE-free synthetic membranes to elucidate the direct role of the $C 2 A B$ domain of the soluble $\mathrm{Ca}^{2+}$-sensor Doc2b. Contacting membranes are often coupled by a Doc $2 \mathrm{~b}$-coated membrane stalk that resists forces up to $600 \mathrm{pN}$ upon bead separation. Stalk formation depends strictly on $\mathrm{Ca}^{2}+$ and phosphatidylserine. Real-time fluorescence imaging shows phospholipid but not content mixing, indicating membrane hemifusion. Thus, Doc2b acts directly on membranes and stabilizes the hemifusion intermediate in this cell-free system. In living cells, this mechanism may co-occur with progressive SNARE complex assembly, together defining $\mathrm{Ca}^{2+}$-secretion coupling.

\footnotetext{
${ }^{1}$ Department of Physics and Astronomy, LaserLab, VU University, De Boelelaan 1085, 1081 HV Amsterdam, The Netherlands. ${ }^{2}$ Department of Functional Genomics and Clinical Genetics, CNCR, NCA, VU University and VU Medical Center, De Boelelaan 1085, 1081 HV Amsterdam, The Netherlands.

${ }^{3}$ Department of Molecular Cell Biology, VU University, De Boelelaan 1085, 1081 HV Amsterdam, The Netherlands. * These authors contributed equally to this work. †These authors jointly supervised this work. Correspondence and requests for materials should be addressed to A.J.G. (email: a.j.a.groffen@vu.nl).
} 
M any biological functions depend on regulated secretion. Secretory events proceed through a hemifusion intermediate in which only the proximal leaflets of the vesicle and plasma membrane have fused, as evidenced by electrophysiological measurements ${ }^{1}$, assays based on phospholipid versus content mixing ${ }^{2-4}$ or on the mixing of phospholipids from the outer and inner membrane leaflet ${ }^{5,6}$. A widely used mechanistic model postulates the occurrence of an hourglass-shaped intermediate or stalk structure ${ }^{7}$. Such structures were indeed observed by X-ray diffraction in preparations of multiple parallel bilayers ${ }^{8}$. Membrane fusion in living cells essentially requires the gradual assembly of the SNARE complex that progresses from a trans- to a cis-conformation ${ }^{9-11}$. The precise timing of secretory events is regulated by signalling pathways that differ between tissues, but almost always converge upon the generation of an intracellular $\mathrm{Ca}^{2+}$ signal. These $\mathrm{Ca}^{2+}$ ions activate $\mathrm{C} 2$ domain-containing proteins such as members of the Synaptotagmin, Doc2 and Ferlin families ${ }^{12}$, which interact with membranes and SNARE proteins to trigger secretion ${ }^{13}$.

Previous work has suggested different roles for $\mathrm{Ca}^{2+}$-sensors in secretion coupling. Genetic ablation of Synaptotagmin-1 (Syt1) - a membrane-anchored protein that causes mixed effects on neurotransmitter release-severely impairs fast $\mathrm{Ca}^{2+}$-induced secretion $^{14,15}$. The direct interaction of Syt1's cytosolic C2 domains with phosphatidylserine (PS) prompted the idea that the $\mathrm{Ca}^{2+}$ sensor could directly act in membrane remodelling ${ }^{16}$. In support of this idea, the tandem C2 domains of Syt1 can induce local curvature by shallow insertion of these domains into the lipid bilayer, and this membrane-bending activity correlates with its activity in enhancing the fusion of SNARE liposomes ${ }^{17,18}$. On the other hand, the lack of Syt1 also causes increased rates of spontaneous release rate in resting synapses ${ }^{14}$. These observations suggested that the sensor could arrest fusion at a late stage in the secretion process until a $\mathrm{Ca}^{2+}$ trigger relieves this inhibitory activity ${ }^{19}$. Mechanistically, such an inhibitory activity was proposed to involve binding of Syt1 to the SNARE complex, thereby preventing fusion to proceed in the absence of a triggering $\mathrm{Ca}^{2+}$ signal (often referred to as 'clamping') ${ }^{20}$.

The fusion-enhancing and clamping mechanisms are not mutually exclusive: it is possible that a sensor both inhibits membrane fusion at low $\mathrm{Ca}^{2+}$ and enhances it after $\mathrm{Ca}^{2+}$ elevation ${ }^{13}$. In some experimental conditions, both activities have been separated ${ }^{21,22}$. For example, in Synaptotagmin-2, a mutation of the SNARE-binding polylysine motif caused a loss of spontaneous release inhibition, whereas the fast $\mathrm{Ca}^{2+}$-evoked secretory response was largely normal ${ }^{22}$. Vice versa, mutation of a $\mathrm{Ca}^{2+}$-binding aspartate $\mathrm{D} 364 \mathrm{~N}$ at the membrane-inserting interface impaired the fast secretory response but left spontaneous release unaffected ${ }^{22}$.

Despite many elegant efforts to resolve the precise mechanism, it remains a challenge to investigate the role of the sensorSNARE and sensor-membrane interactions by mutagenesis because the sensor's residues that mediate SNARE binding also mediate membrane binding ${ }^{23,24}$. To address this issue, we established a new method combining real-time fluorescence imaging, microfluidics and force spectroscopy to study how the $\mathrm{Ca}^{2+}$ sensor Doc2b interacts with two opposing membranes. We focused on the $\mathrm{C} 2 \mathrm{AB}$ fragment of Doc $2 \mathrm{~b}$ (referred to as Doc2b in this study) for several reasons. First, soluble $\mathrm{C} 2 \mathrm{AB}$ fragments of Doc2b and Syt1 are sufficient for $\mathrm{Ca}^{2+}$-dependent membrane fusion in cell-free assays ${ }^{17,18,25}$. In Syt1 null neurons, a $\mathrm{C} 2 \mathrm{AB}$ fragment targeted to the presynaptic plasma membrane using a GAP43 domain effectively rescues fast neurotransmission as detected by electrophysiology ${ }^{18}$. Second, Doc $2 \mathrm{~b}$ is more sensitive to $\mathrm{Ca}^{2+}$ than Syt1 (refs 25,26), allowing to perform our measurements at lower $\mathrm{Ca}^{2+}$, minimizing the risk of forming $\mathrm{Ca}^{2+}$-induced artificial structures previously observed in PS-rich membranes ${ }^{27}$. With this assay, we find that Doc2b triggers the formation of a stable membrane stalk, which allows for lipid but not content mixing. These results show that, in vitro, Doc2b C2-phospholipid interactions are sufficient to induce hemifusion in the absence of SNAREs.

\section{Results}

Doc2b induces the formation of membrane stalks. The geometry of fusing membranes was approximated by coating individual microspheres $(3.84 \mu \mathrm{m}$ diameter; Fig. 1a and Supplementary Fig. 1) with phospholipid bilayers containing 20\% PS and $80 \%$ phosphatidylcholine (PC). Uniform bead coating was confirmed using the fluorescent tracer $\mathrm{N}$-(7-nitrobenz-2-oxa-1,3-diazol-4-yl)1,2-dihexadecanoyl-sn-glycero-3-phosphoethanolamine (NBD-PE) in control samples, as well as by electron microscopy where the thickness was close to that of a single bilayer (Supplementary Fig. 2). The microspheres were manipulated with subnanometre precision in two optical traps enabling force detection with $0.1 \mathrm{pN}$ precision ${ }^{28}$. To deal with the existing variation in the diameter of the beads, the point of membrane contact was established empirically for each bead pair by an automated procedure (see Methods section: 'Approach-and-separation routine' for details). After $5 \mathrm{~s}$ of membrane contact in buffer containing $0.74 \mu \mathrm{M}$ Doc $2 \mathrm{~b}$ and $250 \mu \mathrm{M} \mathrm{Ca}^{2+}$, the beads were separated and membrane stalks were observed (Fig. 1a and Supplementary Fig. 3a). The stalks were visualized by real-time imaging of either NBD-PE (Fig. 1b) or Doc $2 \mathrm{~b}(0.39 \mu \mathrm{M} \quad \mathrm{C} 2 \mathrm{AB}$-enhanced green fluorescent protein (EGFP); Fig. 1c). These experiments show that the membrane
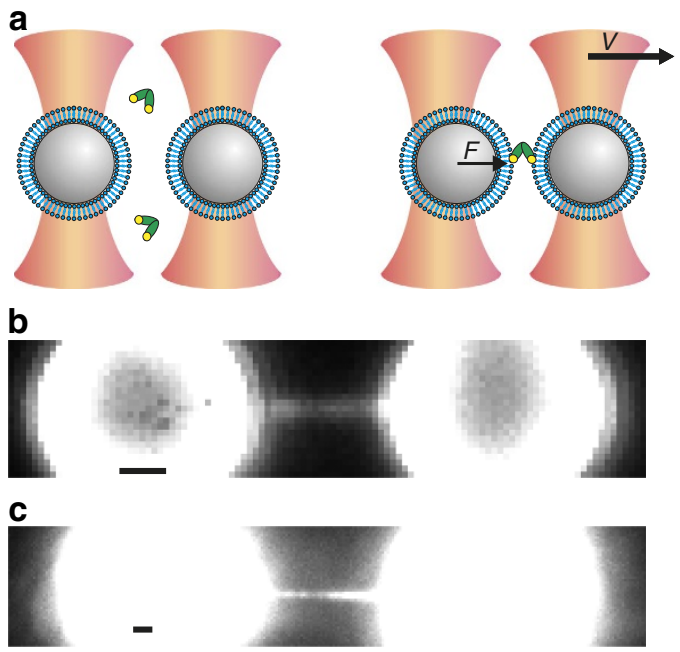

Figure 1 | Principle of combined dual-beam optical trapping and fluorescence microscopy to detect membrane fusion. (a) Schematic (not to scale) of two polystyrene beads (grey) coated with a lipid bilayer (blue) trapped in focused laser beams (orange). Protein fragments comprising Doc $2 b$ (green) were added to the aqueous compartment and could bind phospholipids in the presence of $\mathrm{Ca}^{2+}$ (yellow). Upon bead separation (with constant velocity $v$ ), the force $(F)$ on the left bead was measured. Unless otherwise indicated, experiments were performed at $0.74 \mu \mathrm{M}$ Doc $2 \mathrm{~b}$ with membranes composed of $80 \%$ PC and $20 \%$ PS and in $250 \mu \mathrm{M}$ $\mathrm{Ca}^{2+}$. (b) High-force events were accompanied by the formation of a micrometre-long membrane stalk, revealed by simultaneous fluorescence imaging in the presence of the fluorescent phospholipid NBD-PE. (c) Using unlabelled membranes, a fluorescently tagged Doc2b fragment, $0.39 \mu \mathrm{M}$ C2AB-EGFP, bound efficiently to the entire bilayer including the membrane stalk. Scale bars, $1 \mu \mathrm{m}$. 
including the stalk was completely coated with Doc2b, suggesting that it may help to induce or stabilize the stalk.

Rupture of the membrane stalk requires forces up to $600 \mathrm{pN}$. To quantitatively study the stalk formation process under various conditions, we hypothesized that it should take significant force to rupture a stalk when separating the beads ${ }^{29,30}$. Rupture events can therefore act as readout for stalk formation (Fig. 2a,b and Supplementary Fig. 3). As expected, we observed many interactions between the beads in the presence of all components (Doc $2 \mathrm{~b}, \mathrm{Ca}^{2+}$ and PS). The rupture forces of these interactions exhibited a distribution with a prominent peak around $6 \pm 1 \mathrm{pN}$ $(\mathrm{N}=341$; \pm values indicate s.e.m.) and a long tail with events up to $600 \mathrm{pN}$ ( $N=117$; Fig. $2 \mathrm{c}$ and Supplementary Table 1$)$. In the absence of Doc2b, the rupture forces during bead separation exhibited a distribution with only a peak around $4.18 \pm 0.03 \mathrm{pN}$ $(N=661)$ and lacking the long tail (Fig. 2d and Supplementary Table 1). Based on this observation we consider this low force peak as the background signal in our experiments and the high force tail as indicative of stalk formation.

To discriminate protein-mediated fusion events from background events, we introduced a threshold defined as the mean +4 times the s.d. of the background signal (Supplementary Fig. 4). We chose this relatively high threshold to make sure that the specific interactions are not overwhelmed by the background (Fig. 2c,d and Supplementary Table 1). The average force of the rupture events in the presence of all components was $46 \pm 6 \mathrm{pN}$ in
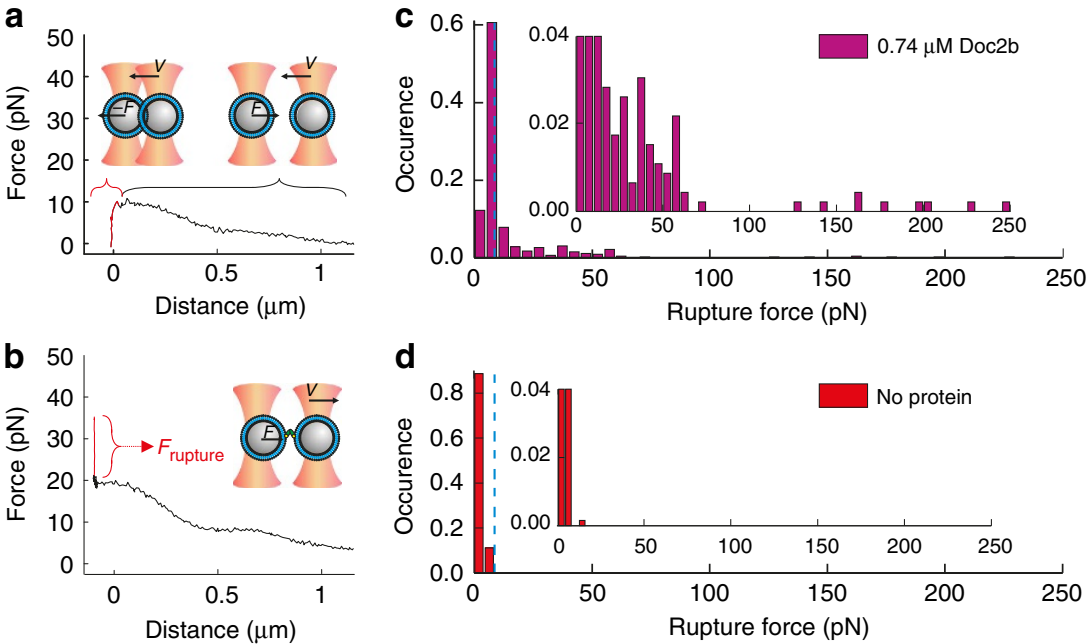

d
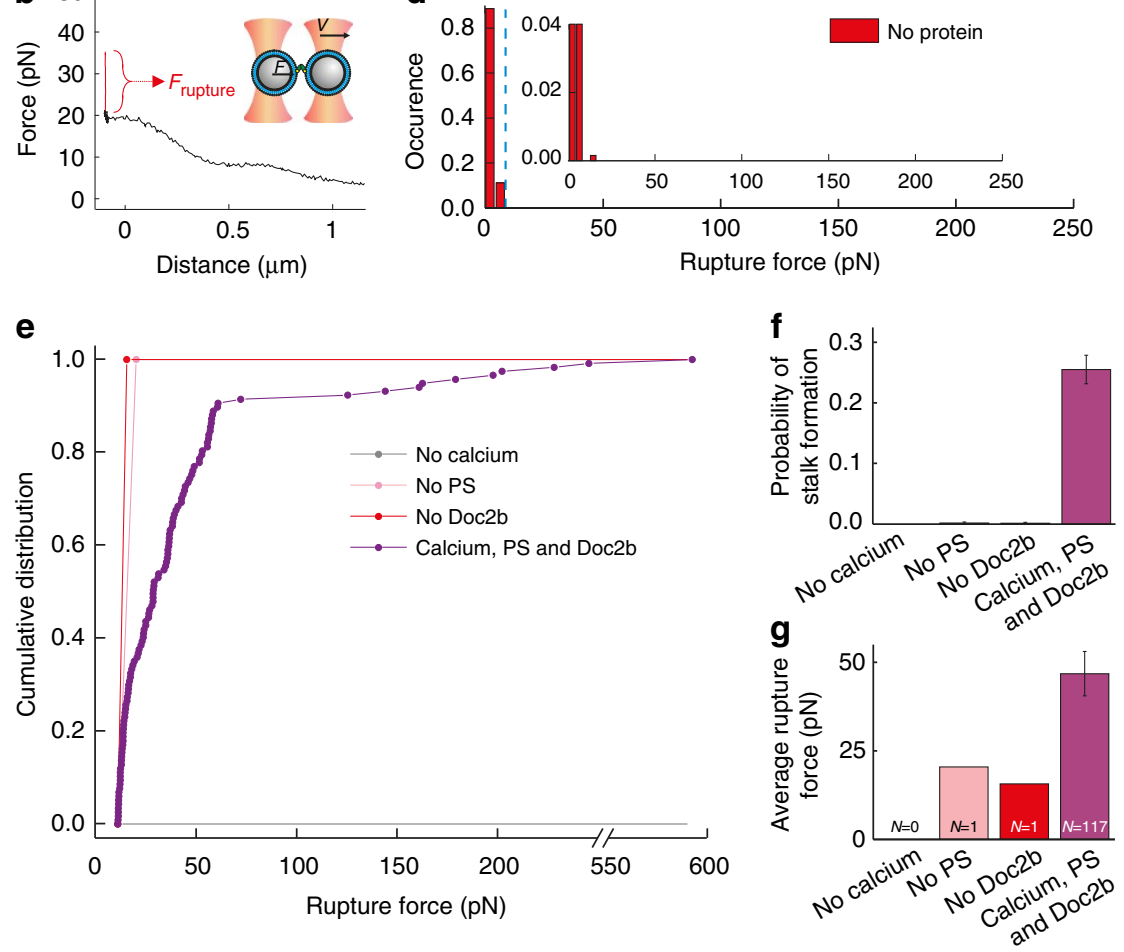

Figure 2 | Doc2b induces stalk formation in a $\mathbf{C a}^{\mathbf{2}+}$ - and PS-dependent manner. (a) Typical force-distance curve in case no membrane stalk was formed. During bead approach, the force initially increases because of interference between the two optical traps. When the beads touch, a drop in the force is recorded as the beads are pushed out of their traps. Retraction speed of the bead was $2 \mu \mathrm{m} \mathrm{s}^{-1}$ in all experiments. (b) Typical force-distance curve in case of a stalk formation event. A clear peak is observed (shown in red) with a corresponding rupture force (indicated in red as $F_{\text {rupture }}$ ) ranging up to $0.6 \mathrm{nN}$. (c) During bead separation in the presence of $20 \%$ PS, $250 \mu \mathrm{M} \mathrm{Ca}^{2+}$ and $0.74 \mu \mathrm{M} \mathrm{Doc} 2 \mathrm{~b}$, a broad distribution of rupture forces values was observed $(N=458)$. Inset: same distribution but with different scaling of the vertical axis. Blue dashed line shows the threshold of $11.3 \mathrm{pN}$ (defined as mean $+4 \times$ s.d., see Supplementary Fig. 4 for details) used to discriminate high-force events. (d) In the absence of Doc $2 \mathrm{~b}$ but the presence of $20 \%$ PS and $250 \mu \mathrm{M} \mathrm{Ca}^{2+}$, only one high-force event was observed out of 661 trials. Inset as in c. (e) Cumulative distributions of rupture events in the presence of $0.74 \mu \mathrm{M}$ Doc2b, $20 \%$ PS and $250 \mu \mathrm{M} \mathrm{Ca}^{2}+$ (purple) or in the absence of $\mathrm{Ca}^{2+}$ (grey), PS (pink) or Doc2b (red). (f) Probability of stalk formation in the absence of $\mathrm{Ca}^{2+}$ (0 events during 291 trials), PS (1 event during 556 trials) or Doc2b (1 event during 661 trials) or in the presence of $0.74 \mu \mathrm{M} \mathrm{Doc} 2 \mathrm{~b}, 20 \%$ $\mathrm{PS}$ and $250 \mu \mathrm{M} \mathrm{Ca}^{2+}(117$ events during 458 trials). Error bars indicate the statistical error in the number of counts. (g) Corresponding rupture forces (mean \pm s.e.m.). 
line with previously reported values of membrane stalk rupture $^{29,30}$ (Fig. $2 \mathrm{~g}$ and Supplementary Table 1). Successive approach-retraction cycles did not significantly change the rupture force (Supplementary Fig. 5). The latter is important because it indicates that the lipid coating of the beads remains intact during the experiment.

Stalk formation requires $\mathrm{Ca}^{2+}$ and PS. Using this method for separating signal from background, we tested if the observed stalk formations required Doc $2 \mathrm{~b}, \mathrm{Ca}^{2+}$ and PS. High-force ruptures were detected during 117 of 458 bead separations in the presence of Doc $2 \mathrm{~b}, \mathrm{Ca}^{2+}$ and PS ( $\left.26 \pm 2 \%\right)$, only in 1 of 661 in the absence of Doc $2 \mathrm{~b}(0.2 \pm 0.2 \%)$, in 0 of 291 in the absence of $\mathrm{Ca}^{2+}$ $(<0.2 \%)$ and in 1 of 556 in the absence of PS in the membrane $(0.2 \pm 0.2 \%$; Fig. $2 \mathrm{e}-\mathrm{f}$ and Supplementary Table 1$)$. Thus, the probability of membrane stalk formation increased more than a hundred-fold in the presence of $\mathrm{Ca}^{2+}$, PS and Doc2b, indicating that stalk formation is a specific process, which involves $\mathrm{C} 2-$ phospholipid interactions ${ }^{31}$.

Membrane hemifusion. The Doc2b-covered membrane stalk could represent various molecular configurations: it could consist of (i) two individual membranous tubes connected by a protein bridge; (ii) a structure with a continuous outer membrane leaflet (hemifusion) or (iii) a continuous membrane tube that connects the lumen of the two membrane-coated beads (full fusion). To distinguish between these scenarios, we conducted experiments that probed either lipid or content mixing. To test lipid mixing, we captured one bead coated with $70 \%$ PC/20\% PS/10\% fluorescent NBD-PE and a second bead coated with $80 \% \mathrm{PC} / 20 \%$ PS. After formation of a membrane stalk, the stalk and unlabelled bead started to fluoresce (Fig. 3a,c,e). Hence, we concluded that the stalk consists of a continuous phospholipid structure as expected for hemifusion or full fusion. To test if the membrane stalk also allows content mixing, we captured a bead with soluble fluorescein, a 389-Da fluorophore small enough to diffuse through a fusion pore, in the luminal compartment between the membrane and bead (Supplementary Fig. 1 for production method), whereas the lumen of the other trapped bead was not fluorescent. Upon Doc2b-induced formation of a membrane stalk, the fluorescence signal of the unlabelled bead and the stalk did not increase (Fig. 3b,d,e). We performed a number of controls to make sure that the absence of fluorescein in the unlabelled bead was not caused by immobilized fluorescein: (i) confocal bead imaging and fluorescence recovery after photobleaching experiments showed that the fluorescein can freely diffuse within the luminal compartment (Fig. 4a) and NBD can freely diffuse through the lipid bilayer (Fig. 4a), and that, upon membrane disruption by $0.02 \%$ Triton X-100, the fluorescein leaked into the a

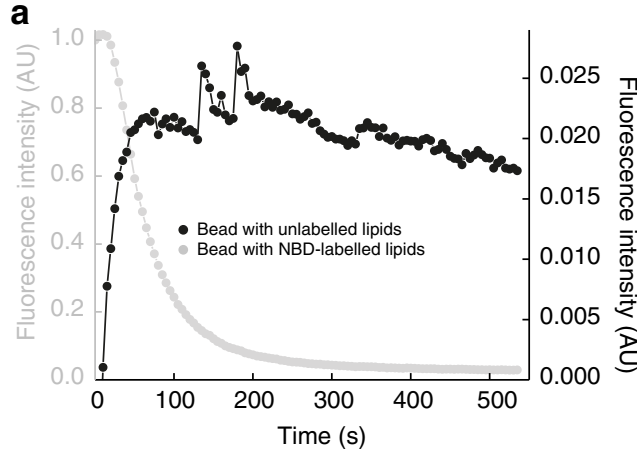

b

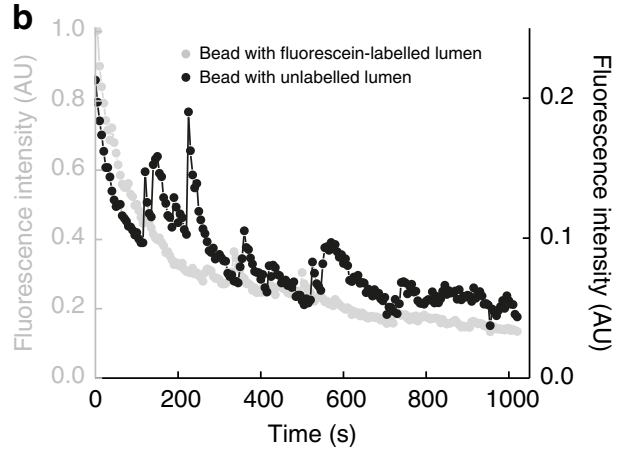

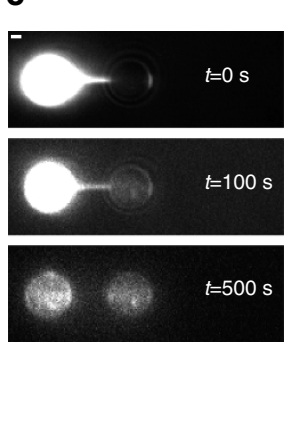

d

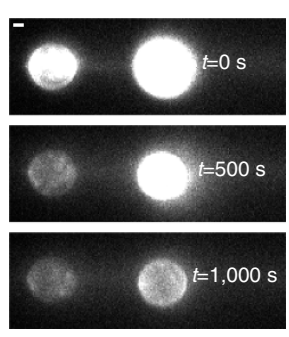

e

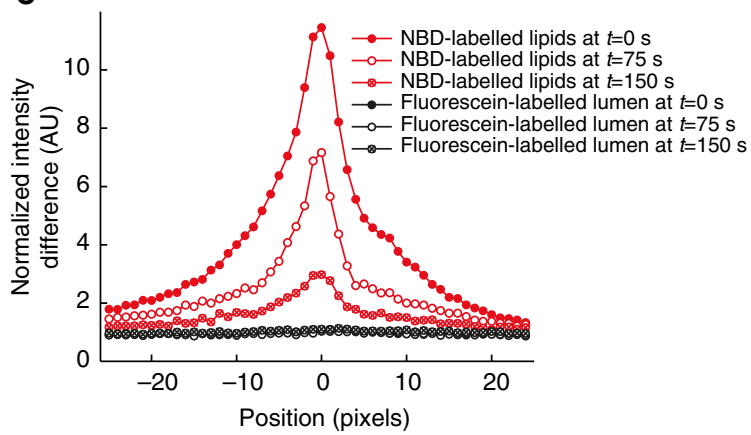

f

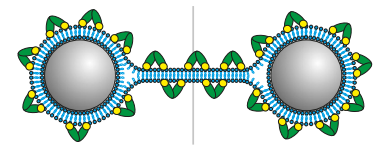

Figure 3 | Membrane hemifusion. (a) In the presence of fluorescent NBD-PE in a single membrane-coated bead, membrane stalk formation was accompanied by a strong fluorescence increase in the unlabelled membrane and a concurrent decrease in the labelled membrane. This phospholipid mixing indicates that either hemifusion or full membrane fusion occurred. Fluorescence intensities were normalized with respect to the initial intensity on the NBDlabelled bead. Even though stalk formation happens readily, to track lipid continuity and content mixing we require long-lived membrane stalks ( $>100 \mathrm{~s}$ ). We measured two of those events. The small peaks in the signal are due to autofluorescent impurities in the buffer flow. (b) To distinguish between hemifusion and full fusion, we tested if membrane stalk formation allowed content mixing by coating the beads with nonfluorescent PC/PS liposomes while one of the beads was loaded with fluorescein in the liposomal lumen. On a timescale of 1,000 s, we did not detect a fluorescence increase in the unlabelled bead or membrane stalk, indicative for hemifusion. The decrease in fluorescence of the fluorescein-labelled bead is due to photobleaching. Fluorescence intensities were normalized with respect to the initial intensity on the fluorescein-labelled bead. In total, four of these events were measured. (c)

Fluorescence images of bead pair in $\mathbf{a}$ at three different time points. Scale bar, $1 \mu \mathrm{m}$. (d) Fluorescence images of bead pair in $\mathbf{b}$ at three different time points. Scale bar, $1 \mu \mathrm{m}$. (e) Fluorescence intensity profiles along the dashed line indicated in $\mathbf{f}$ at three different time points. Intensities were averaged over 20 pixels perpendicular to the dashed line and normalized to the background signal. (f) Schematic representation of the hemifused configuration with Doc $2 b$ bound to the membrane surface. 
a

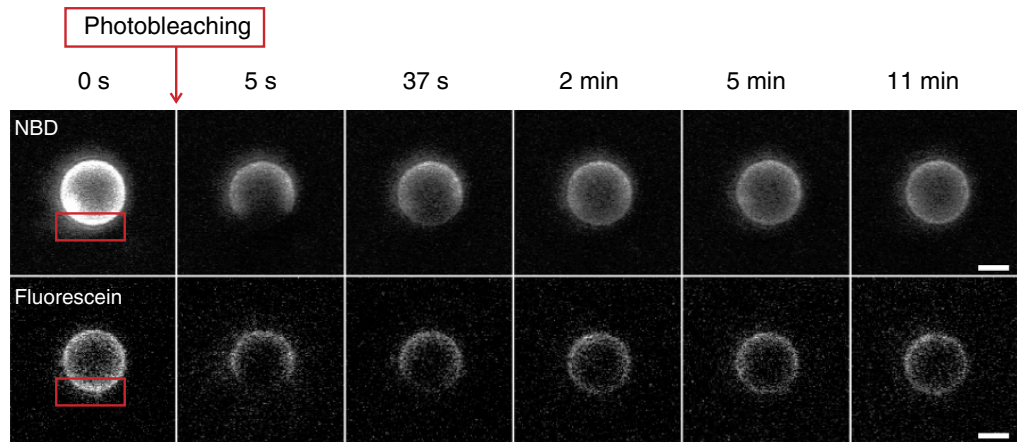

b

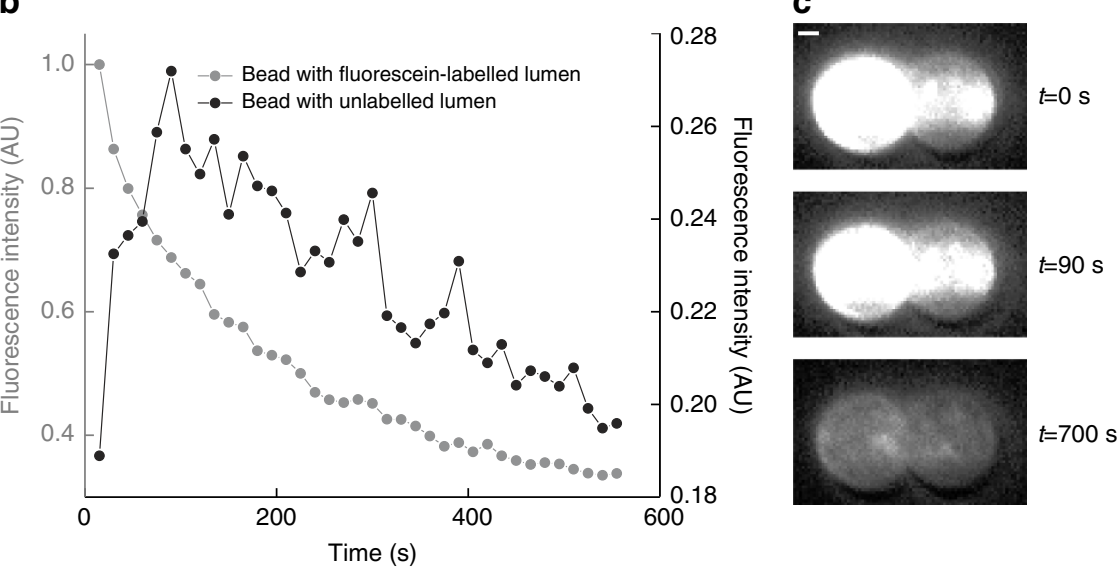

Figure 4 | Fluorescent labeling of either lipids or luminal compartment provides a probe for detecting membrane remodeling, hemifusion of fully fused states. (a) Membrane-coated beads with lipids labelled with NBD-PE or with fluorescein in the luminal compartment were analysed by confocal microscopy before $(t=0 \mathrm{~s})$ and after $(t=5 \mathrm{~s})$ photobleaching of the region indicated by the red rectangle. To assess fluorescence recovery after photobleaching, images were acquired at various times after bleaching. From the fact that the fluorescence intensity recovers after time, we conclude that the fluorophores are free to diffuse through the membrane (in the case of NBD) or within the luminal compartment (for fluorescein). Data shown are representative example from two (in the case of NBD) and ten (in the case of fluorescein) experiments. Scale bar, $2 \mu \mathrm{m}$. (b) When two beads, of which one has a fluorescein-labelled lumen, are brought in contact in the presence of $5 \mathrm{mM} \mathrm{CaCl}$ in the buffer for at least 1 min, membrane fusion occurred. This fusion was accompanied by an increase of fluorescence signal in the bead that was originally dark (black data set), proving that content mixing, indicative of full membrane fusion, has occurred. The decrease in fluorescence intensity in the grey data set is due to photobleaching. Similar results were obtained in three independent experiments. (c) Fluorescence images of bead pair in $\mathbf{b}$ at three different time points. Note that $t=0 \mathrm{~s}$ is the time at which fluorescence acquisition was started. The initial intensity on the bead (top panel) is thus partially caused by autofluorescence of the beads and partially by content that has already diffused to the other bead. Scale bar, $1 \mu \mathrm{m}$.

surrounding buffer (Supplementary Fig. 6); (ii) we showed using $\mathrm{Ca}^{2+}$-induced membrane fusion that it is possible to detect content mixing with our method (Fig. 4b,c). Hence, the absence of luminal content mixing we observe in the presence of Doc2b demonstrates that the membrane stalk does not contain a fusion pore. Thus, Doc2b directly induces hemifusion in the absence of SNAREs (Fig. 3f).

To confirm Doc2b dose dependence, rupture force profiles were measured in the presence of $0-0.74 \mu \mathrm{M}$ Doc $2 \mathrm{~b}$ (Fig. 5a). The hemifusion probability increased drastically from negligible to $26 \pm 2 \%$ (Fig. 5b and Supplementary Table 1). We could only reliably determine the average rupture for concentrations above $0.15 \mu \mathrm{M}$ where it increased from $18 \pm 4$ to $47 \pm 6 \mathrm{pN}$ (Fig. $5 \mathrm{c}$ and Supplementary Table 1).

\section{Discussion}

The dose-dependent increase in the probability of stalk formation suggests that in our in vitro situation, Doc $2 \mathrm{~b}$ binding primarily lowers the energy barrier for hemifusion, whereas the increase in rupture force suggests a stabilization of the membrane stalk due to protein coverage. In previous studies with atomic force microscopy, Syt1-membrane interactions ${ }^{30}$ yielded rupture forces in the similar range of $72-122 \mathrm{pN}$ (at a $\sim 20$ times higher loading rate). Note that in these experiments the molecular arrangement was different because of the immobilization of glutathione $S$-transferase (GST)-C2AB on a cantilever. In another study, atomic force microscopy tips were coated with lipid bilayers to study Synapsin-1-mediated interactions between opposing membranes ${ }^{29}$. At a retraction velocity of $80-400 \mathrm{~nm} \mathrm{~s}^{-1}$, this yielded rupture forces up to $3 \mathrm{nN}$, which corresponds well with our highest observed force of $0.6 \mathrm{nN}$.

To conclude, our data suggest that Doc $2 \mathrm{~b}$ by itself can promote hemifusion in a manner that depends on $\mathrm{Ca}^{2+}$-dependent binding to PS in the membrane. This activity likely relates to the phenomenon that $\mathrm{C} 2$ domain insertion induces membrane curvature, as was previously shown for the $\mathrm{C} 2 \mathrm{AB}$ fragments of Syt1 and Doc2b, which caused liposome tubulation in cell-free preparations $s^{17,25,32}$. In turn, membrane curvature is predicted to strongly affect the energy state of the hemifusion intermediate according to theoretical models of stalk formation ${ }^{33}$. Together, this suggests that Doc2b contributes to $\mathrm{Ca}^{2+}$-dependent secretion by lowering the energy barrier of hemifusion through direct interaction with PS. This finding may also shed light on an open question regarding the mechanism of Doc2b-dependent enhancement of spontaneous neurotransmitter release in 
a

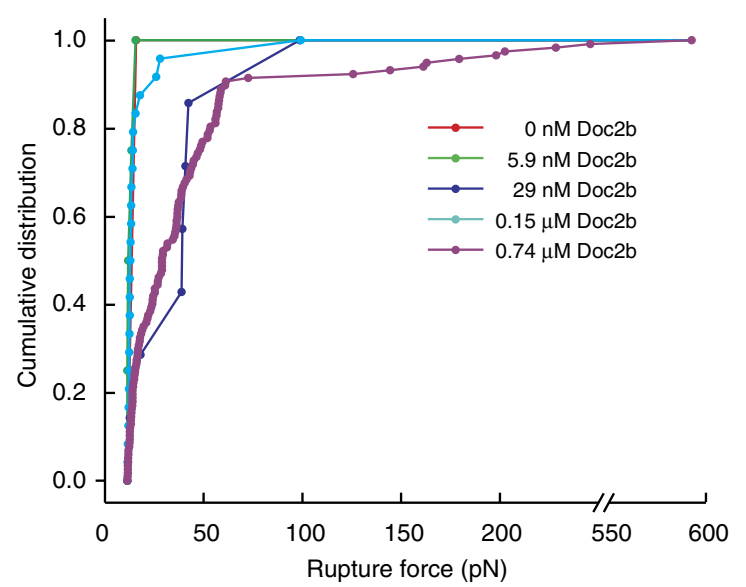

b

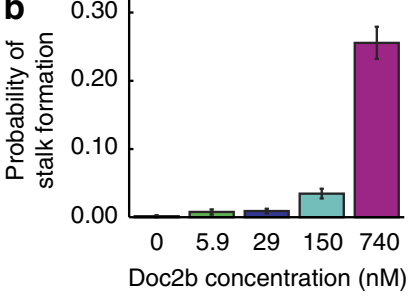

C

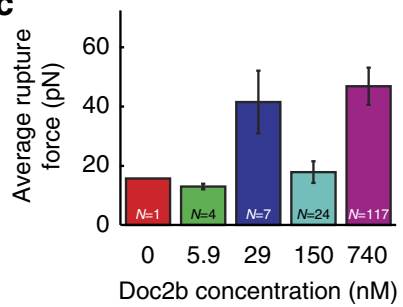

Figure 5 | Doc2b concentration dependence of membrane stalk formation. (a) Cumulative distributions, (b) stalk formation probabilities at $0 \mathrm{nM}$ (1 event during 661 trials), $5.9 \mathrm{nM}$ (4 events out of 521 trials), $29 \mathrm{nM}$ ( 7 events out of 777 trials), $0.15 \mu \mathrm{M}$ ( 24 events out of 694 trials) and $0.74 \mu \mathrm{M}$ ( 117 events out of 458 trials) Doc2b. Error bars indicate the statistical error in the number of counts. (c) Average \pm s.e.m. of rupture forces at the same Doc2b concentrations observed during bead separation in the presence of $20 \%$ PS and $250 \mu \mathrm{M} \mathrm{Ca}^{2+}$. Numbers of above-threshold events are indicated in the graph. A strong increase in the probability of stalk formation was observed as a function of Doc $2 b$ concentration.

neurons ${ }^{25,34}$. Although our in vitro data suggest a direct $\mathrm{Ca}^{2+}$ sensing role by Doc2b in membrane fusion, the mechanism in living systems is likely more complex and additional interactions of Doc2b with SNARE proteins ${ }^{25}$, Munc18 (ref. 35) and Munc13 (ref. 36) must also be considered. In support of this idea, mutations in Doc2b that inhibit SNARE binding, inhibit the fusogenic activity of SNARE-containing liposomes partially (but not completely) ${ }^{25}$. As $\mathrm{C} 2 \mathrm{AB}$ domains and their phospholipid interaction surfaces are highly conserved between $\mathrm{Ca}^{2}{ }^{+}$-sensors, a similar activity is likely to regulate many secretory processes in the body.

Finally, our method may aid the discovery of membrane fusion mechanisms. Liposome fusion assays have yielded tremendous insights into SNARE-mediated membrane fusion ${ }^{9}$ but do not allow detection of the SNARE-independent effects of $\mathrm{Ca}^{2+}$ (ref. 37). Our unique combination of force spectroscopy with fluorescence microscopy enables both direct quantification and visualization of fusion events, while at the same time providing direct evidence for possible lipid and content mixing.

\section{Methods}

Bead coating. 1,2-Dioleoyl-sn-glycero-3-phosphocholine and 1,2-dioleoyl-sn-glycero-3-phospho-L-serine were acquired from Avanti Polar Lipids as solutions in chloroform. NBD-PE was acquired from Invitrogen as chlorophorm solution. The protocol for coating beads with lipids was adapted from previous work ${ }^{38}$. Polystyrene nonporous beads of diameter $3.84 \mu \mathrm{m} \pm 4 \%$ (manufacturer's specifications) were acquired from Spherotech. For each coating procedure, the beads were washed three times in milliQ and sonicated shortly. Liposomes were prepared by mixing the lipids from chloroform stocks in desired proportion, extensively drying the mix under a nitrogen gas stream, and reconstituting the lipids in milliQ to a final concentration of $1 \mathrm{mg} \mathrm{ml}^{-1}$. The solution was vortexed to form giant multilamellar vesicles and then sonicated on ice $(8$ times for $6 \mathrm{~s}$, with at least 1 min interval between each sonication) to produce small unilamellar vesicles. To get rid of the residual multilamellar vesicles, the solution was centrifuged at $21,000 \mathrm{~g}$ for $30 \mathrm{~min}$ at $4^{\circ} \mathrm{C}$. The supernatant was mixed with beads, with addition of $\mathrm{CaCl}_{2}$ to $3 \mathrm{mM}$, and incubated for $16 \mathrm{~h}$ at $4{ }^{\circ} \mathrm{C}$ with gentle continuous mixing to keep the beads in suspension. The beads were washed (by spinning at $900 \mathrm{~g}$ and gently resuspending) in buffer 1 (25 mM HEPES, pH 7.4, $200 \mathrm{mM} \mathrm{NaCl}, 1 \mathrm{mM}$ tris(2-carboxyethyl)phosphine (TCEP), $5 \mathrm{mM}$ EDTA), then buffer 2 (25 mM HEPES, $\mathrm{pH} 7.4,100 \mathrm{mM} \mathrm{NaCl}, 1 \mathrm{mM}$ TCEP, $\left.0.25 \mathrm{mM} \mathrm{CaCl}_{2}\right)$ and then in buffer $3(25 \mathrm{mM}$ HEPES, pH 7.4, $25 \mathrm{mM} \mathrm{NaCl}, 1 \mathrm{mM}$ TCEP and $0.25 \mathrm{mM} \mathrm{CaCl}_{2}$ ). The coated beads were stored at $4{ }^{\circ} \mathrm{C}$ and used within 5 days.

To monitor the bead-coating procedure, we prepared in parallel a sample with addition of $1.5 \%$ fluorescent NBD-PE. These beads were imaged in solution by confocal microscopy on a LSM510 meta system (Carl Zeiss) with excitation at $488 \mathrm{~nm}$ (emission $514 \mathrm{~nm}$ ) with a $\times 63$ oil objective (numerical aperture $=1.3$ ). In addition, we prepared a batch of beads containing $10 \%$ NBD-PE in the membrane coating for the imaging experiments (Figs 1b,3a,c,e and 4a and Supplementary Figs $2 \mathrm{a}$ and 6). As stalk formation was observed with (Fig. 1b) and without NBDPE (Fig. 1c) and similar rupture forces were measured under both conditions, we concluded that the addition of NBD-PE does not have a significant effect on stalk formation. For the experiment shown in Figures 3b,d,e and 4 and Supplementary Fig. 6, we used fluorescein-containing beads. To prepare these beads, the procedure was the same as above except that the dried phospholipids were suspended in a solution containing $250 \mu \mathrm{M}$ fluorescein-5-isothiocyanate (Molecular Probes) dissolved in $10 \mathrm{mM}$ Tris, $\mathrm{pH} 7.4$, leading to the inclusion of fluorescein in the lumen of the liposomes and, ultimately, of the membrane-covered beads (see Supplementary Fig. 1 for details).

Recombinant proteins. Recombinant Doc $2 \mathrm{~b} \mathrm{C} 2 \mathrm{AB}$ (rat, amino-acid residues 115-412) was subcloned into the pGEX vector (GE Healthcare), and transformed into E. coli BL21 DE3 strain for expression. To express a fusion protein of Doc2b aa115-412 and EGFP, the 231-bp EcoR1-NotI fragment of pGEX-GS-C2AB was replaced with the 989-bp EcoR1-NotI fragment of pEGFP-N2-Doc2b (ref. 39). Cultures in LB medium supplemented with $200 \mu \mathrm{g} \mathrm{ml}^{-1}$ ampicillin were grown to $\mathrm{OD}_{600}=0.6-0.8$ and gene expression was induced with $0.5 \mathrm{mM} \mathrm{IPTG}$ at $37^{\circ} \mathrm{C}$ for $4 \mathrm{~h}$. Bacterial pellets were lysed in $300 \mathrm{mM} \mathrm{NaCl}, 50 \mathrm{mM}$ Tris, $\mathrm{pH} 7.5,10 \mathrm{mM}$ EDTA, $1 \mathrm{mg} \mathrm{ml}^{-1}$ lysozyme and protease inhibitor cocktail, sonicated five times for $15 \mathrm{~s}$, incubated in the presence of $1 \%$ Triton X-100 and centrifuged for $30 \mathrm{~min}$ at $10,000 \mathrm{~g}$ to remove insoluble cell debris. GST-tagged C2 domains were isolated from bacterial lysates by affinity purification with glutathione agarose (Sigma), washed extensively in high-salt buffer $(300 \mathrm{mM} \mathrm{NaCl}, 50 \mathrm{mM}$ Tris $\mathrm{pH} 7.4,10 \mathrm{mM}$ EDTA). The beads were then loaded into a disposable column where the buffer was replaced with DNA/RNA digestion buffer $(150 \mathrm{mM} \mathrm{NaCl}, 50 \mathrm{mM}$ Tris $\mathrm{pH} 7.4$ and $1.4 \mathrm{mM} \mathrm{MgCl}_{2}$ ), followed by incubation with $1 \mathrm{mg} \mathrm{ml}^{-1}$ each of DNAseI (Roche) and RNAse A (Boehringer Mannheim) for $20 \mathrm{~min}$ at $37^{\circ} \mathrm{C}$. The beads were washed again five times with high-salt buffer and three times with low-salt buffer $(150 \mathrm{mM}$ $\mathrm{NaCl}$ and $50 \mathrm{mM}$ Tris, $\mathrm{pH}$ 7.4). C2AB domains were then cleaved from GST using thrombin (Serva) for $16 \mathrm{~h}$ at $4{ }^{\circ} \mathrm{C}$. Protein concentration was determined by SDSPAGE and SYPRO Ruby staining, using bovine serum albumin (Sigma) as a standard. Proteins were diluted to the indicated concentration in buffer 3 as specified above. All buffers were filtered through a $0.2-\mu \mathrm{m}$ pore size and stored at $4{ }^{\circ} \mathrm{C}$ for no longer than a week.

Optical trapping. All measurements were performed at room temperature. The optical tweezers setup used was previously described in detail ${ }^{40}$. Briefly, the combined optical trapping and fluorescence-microscopy setup was custom-built around a Nikon inverted microscope, equipped with a 1,064-nm trapping laser. The laser beam is split into two perpendicularly polarized beams using a half-wave plate and a polarizing beam splitter to produce two independent traps that can be used to manipulate two individual beads. The position of the first trap can be controlled by steering one of the telescope lenses. The two beams are combined using another polarizing beam splitter and coupled into a water-immersion objective. Forcedetection on one of the beads is then achieved by collecting the transmitted light in an oil-immersion condenser, rejecting the unwanted polarization using a polarizing beam splitter and imaging on a position-sensitive diode. 
Using a blue light-emitting diode to illuminate the sample, bright-field images of the trapped beads were obtained on a CCD camera allowing real-time determination of the bead-to-bead distance. For the wide-field fluorescence microscopy, NBD-PE, EGFP and fluorescein were excited by a $491-\mathrm{nm}$ laser, coupled into the microscope and imaged on an EMCCD camera. A custom-made glass multichannel laminar flow cell was used to obtain parallel flow and to facilitate rapid buffer exchange between channels containing lipid-coated beads, buffer and protein.

Approach-and-separation routine. When the beads were kept far apart ( $>2 \mu \mathrm{m})$, the measured force was zero. When the beads were moved very close together $(<0.5 \mu \mathrm{m})$ one optical trap exerted a force on the bead in the other trap in the range of $5-15 \mathrm{pN}$ (see Fig. $2 \mathrm{a}$ for details); an effect that was independent of lipid coating on the beads. Because of the intrinsic variation in bead size (standard deviation $4 \%$ as indicated by the manufacturer), an automated stepping procedure was employed for each bead pair to determine the distance at which specific Doc2b-induced interactions occurred (Supplementary Fig. 3).This stepwise exploration was stopped as soon as a rupture force of at least $5 \mathrm{pN}$ was detected during bead separation. The approach-and-retraction cycle was then repeated 20-160 times using the same distance (Supplementary Fig. 3b, arrows indicate rupture events). During many repeated cycles there was no detectable trend in the observed force values, suggesting that the membrane coating remained intact (Supplementary Fig. 5). The speed at which the bead is retracted is known to influence rupture forces. Therefore, a constant trap speed of $2 \mu \mathrm{m} \mathrm{s}^{-1}$ was used in all experiments.

Data acquisition and analysis. Data analysis was performed using customwritten software in LabView (National Instruments) to extract force data at a frequency of $50 \mathrm{kHz}$. In post-processing, peak heights were determined from force traces (such as Supplementary Fig. 3b) by subtracting the baseline (average of force-values before the peak) from the maximum force (at the top of the peak). To discriminate between stalk formation events and unsuccessful events, we used a single threshold, determined by performing a Gaussian fit to the major peak in the rupture force distribution containing all observed peaks (Supplementary Fig. 4). We then used the mean value plus four times the standard deviation of this peak as a threshold, resulting in a threshold value of $11.3 \mathrm{pN}$.

Electron microscopy. Polystyrene beads were coated with lipid membrane as described above, and incubated with protein and $\mathrm{Ca}^{2+}$-containing buffers as indicated. The beads were fixed and contrasted by $1 \%$ osmium-tetraoxide (EMS) before embedding in $10 \%$ gelatine droplets for cryo-sectioning. $70 \mathrm{~nm}$ sections were collected on carbon/formvar-coated mesh grids and stabilized by methylcellulose in the presence of $0.4 \%$ uranyl acetate when indicated. Transmission electron microscopy images were collected on a JEOL1010 TEM (JEOL) with Morada CCD camera (Olympus Soft Imaging Solutions) at $\times 80,000$ magnification. The thickness of the electron-dense coating was quantitated in iTEM software (Olympus).

\section{References}

1. Melikyan, G. B., Abidor, I. G., Chernomordik, L. V. \& Chailakhyan, L. M. Electrostimulated fusion and fission of bilayer lipid membranes. Biochim. Biophys. Acta - Biomembr 730, 395-398 (1983).

2. Kemble, G. W., Danieli, T. \& White, J. M. Lipid-anchored influenza hemagglutinin promotes hemifusion, not complete fusion. Cell 76, 383-391 (1994).

3. Wang, T., Smith, E. A., Chapman, E. R. \& Weisshaar, J. C. Lipid mixing and content release in single-vesicle, SNARE-driven fusion assay with 1-5 ms resolution. Biophys. J. 96, 4122-4131 (2009).

4. Kyoung, M. et al. In vitro system capable of differentiating fast $\mathrm{Ca}^{2+}$-triggered content mixing from lipid exchange for mechanistic studies of neurotransmitter release. Proc. Natl Acad. Sci. USA 108, E304-E313 (2011).

5. Lu, X., Xu, Y., Zhang, F. \& Shin, Y. K. Synaptotagmin I and Ca2+ promote half fusion more than full fusion in SNARE-mediated bilayer fusion. FEBS Lett. 580, 2238-2246 (2006).

6. Liu, T., Wang, T., Chapman, E. R. \& Weisshaar, J. C. Productive hemifusion intermediates in fast vesicle fusion driven by neuronal SNAREs. Biophys. J. 94, 1303-1314 (2008)

7. Markin, V. S., Kozlov, M. M. \& Borovjagin, V. L. On the theory of membrane fusion. The stalk mechanism. Gen. Physiol. Biophys. 3, 361-377 (1984).

8. Yang, L. \& Huang, H. W. Observation of a membrane fusion intermediate structure. Science 297, 1877-1879 (2002).

9. Weber, T. et al. SNAREpins: minimal machinery for membrane fusion. Cell 92, 759-772 (1998).

10. Stein, A., Weber, G., Wahl, M. C. \& Jahn, R. Helical extension of the neuronal SNARE complex into the membrane. Nature 460, 525-528 (2009).

11. Gao, Y. et al. Single reconstituted neuronal SNARE complexes zipper in three distinct stages. Science 337, 1340-1343 (2012).

12. Walter, A. M., Groffen, A. J., Sørensen, J. B. \& Verhage, M. Multiple Ca2 + sensors in secretion: teammates, competitors or autocrats? Trends Neurosci. 34, 487-497 (2011).
13. Chicka, M. C., Hui, E., Liu, H. \& Chapman, E. R. Synaptotagmin arrests the SNARE complex before triggering fast, efficient membrane fusion in response to Ca2 +. Nature Struct. Mol. Biol. 15, 827-835 (2008).

14. Littleton, J. T., Stern, M., Schulze, K., Perin, M. \& Bellen, H. J. Mutational analysis of Drosophila synaptotagmin demonstrates its essential role in $\left.\mathrm{Ca}^{2+}\right)$ activated neurotransmitter release. Cell 74, 1125-1134 (1993).

15. Geppert, M. et al. Synaptotagmin I: a major $\mathrm{Ca} 2+$ sensor for transmitter release at a central synapse. Cell 79, 717-727 (1994).

16. Brose, N., Petrenko, A. G., Südhof, T. C. \& Jahn, R. Synaptotagmin: a calcium sensor on the synaptic vesicle surface. Science 256, 1021-1025 (1992).

17. Martens, S., Kozlov, M. M. \& McMahon, H. T. How synaptotagmin promotes membrane fusion. Science 316, 1205-1208 (2007).

18. Hui, E., Johnson, C. P., Yao, J., Dunning, F. M. \& Chapman, E. R. Synaptotagmin-mediated bending of the target membrane is a critical step in $\mathrm{Ca}(2+)$-regulated fusion. Cell 138, 709-721 (2009).

19. Popov, S. V. \& Poo, M. M. Synaptotagmin: a calcium-sensitive inhibitor of exocytosis? Cell 73, 1247-1249 (1993).

20. Söllner, T., Bennett, M. K., Whiteheart, S. W., Scheller, R. H. \& Rothman, J. E. A protein assembly-disassembly pathway in vitro that may correspond to sequential steps of synaptic vesicle docking, activation, and fusion. Cell 75, 409-418 (1993).

21. Littleton, J. T., Stern, M., Perin, M. \& Bellen, H. J. Calcium dependence of neurotransmitter release and rate of spontaneous vesicle fusions are altered in Drosophila synaptotagmin mutants. Proc. Natl Acad. Sci. USA 91, 10888-10892 (1994).

22. Kochubey, O. \& Schneggenburger, R. Synaptotagmin increases the dynamic range of synapses by driving $\mathrm{Ca}^{2+}$-evoked release and by clamping a nearlinear remaining $\mathrm{Ca}^{2+}$ sensor. Neuron 69, 736-748 (2011).

23. Rickman, C. et al. Synaptotagmin interaction with the syntaxin/SNAP-25 dimer is mediated by an evolutionarily conserved motif and is sensitive to inositol hexakisphosphate. J. Biol. Chem. 279, 12574-12579 (2004).

24. Bai, J., Tucker, W. C. \& Chapman, E. R. PIP2 increases the speed of response of synaptotagmin and steers its membrane-penetration activity toward the plasma membrane. Nature Struct. Mol. Biol. 11, 36-44 (2004).

25. Groffen, A. J. et al. Doc2b is a high-affinity Ca2 + sensor for spontaneous neurotransmitter release. Science 327, 1614-1618 (2010).

26. Groffen, A. J. A., Friedrich, R., Brian, E. C., Ashery, U. \& Verhage, M. DOC2A and $\mathrm{DOC} 2 \mathrm{~B}$ are sensors for neuronal activity with unique calcium-dependent and kinetic properties. J. Neurochem. 97, 818-833 (2006).

27. Ginsberg, L. Does $\mathrm{Ca} 2+$ cause fusion or lysis of unilamellar lipid vesicles? Nature 275, 758-760 (1978).

28. Gross, P. et al. Quantifying how DNA stretches, melts and changes twist under tension. Nat. Phys 7, 731-736 (2011).

29. Pera, I., Stark, R., Kappl, M., Butt, H.-J. \& Benfenati, F. Using the atomic force microscope to study the interaction between two solid supported lipid bilayers and the influence of synapsin I. Biophys. J. 87, 2446-2455 (2004).

30. Takahashi, H., Shahin, V., Henderson, R. M., Takeyasu, K. \& Edwardson, J. M. Interaction of synaptotagmin with lipid bilayers, analyzed by single-molecule force spectroscopy. Biophys. J. 99, 2550-2558 (2010).

31. Rizo, J. \& Sudhof, T. C. C2-domains, Structure and Function of a Universal Ca2 + -binding Domain. J. Biol. Chem. 273, 15879-15882 (1998).

32. Campelo, F., McMahon, H. T. \& Kozlov, M. M. The hydrophobic insertion mechanism of membrane curvature generation by proteins. Biophys. J. 95, 2325-2339 (2008).

33. Markin, V. S. \& Albanesi, J. P. Membrane fusion: stalk model revisited. Biophys. J. 82, 693-712 (2002).

34. Pang, Z. P. et al. Doc2 supports spontaneous synaptic transmission by a $\mathrm{Ca}(2+)$-independent mechanism. Neuron 70, 244-251 (2011).

35. Verhage, M. et al. DOC2 proteins in rat brain: complementary distribution and proposed function as vesicular adapter proteins in early stages of secretion. Neuron 18, 453-461 (1997).

36. Orita, S. et al. Physical and functional interactions of Doc2 and Munc13 in Ca2 + -dependent exocytotic machinery. J. Biol. Chem. 272, 16081-16084 (1997)

37. Kyoung, M., Zhang, Y., Diao, J., Chu, S. \& Brunger, A. T. Studying calciumtriggered vesicle fusion in a single vesicle-vesicle content and lipid-mixing system. Nat. Protoc. 8, 1-16 (2013).

38. Baksh, M. M. et al. Neuronal activation by GPI-linked neuroligin-1 displayed in synthetic lipid bilayer membranes. Langmuir 21, 10693-10698 (2005).

39. Groffen, A. J. A. et al. $\mathrm{Ca}(2+)$-induced recruitment of the secretory vesicle protein DOC2B to the target membrane. J. Biol. Chem. 279, 23740-23747 (2004).

40. Gross, P., Farge, G., Peterman, E. J. G. \& Wuite, G. J. L. Combining optical tweezers, single-molecule fluorescence microscopy, and microfluidics for studies of DNA-protein interactions. Methods Enzymol. 475, 427-453 (2010).

\section{Acknowledgements}

We thank Dr Maarten Noom for his help with preliminary experiments and Rien Dekker for technical assistance. This study was supported by the Netherlands Organization for Scientific Research (grant numbers 91111009,021002077 and a VICI grant to G.J.L.W.), 
the Netherlands Organization for Health Research and Development (ZonMW, 91113022), an ERC starting grant and a FOM program grant to G.J.L.W.

\section{Author contributions}

A.G., N.L., G.J.L.W and A.J.G. designed the study. Experimental contributions were as follows: bead coating and protein purification by A.G., A.J.G. and D.B.; optical trapping experiments by I.B., A.G. and N.L., fluorescence recovery after photobleaching by A.J.G., EM measurements by J.R.T. vanW. Data analysis software was written by N.L. Data analysis was performed by I.B., A.G., N.L. and A.J.G. Manuscript was written by I.B., A.G., G.J.L.W. and A.J.G. and edited by all authors.

\section{Additional information}

Supplementary Information accompanies this paper at http://www.nature.com/ naturecommunications
Competing financial interests: The authors declare no competing financial interests.

Reprints and permission information is available online at http://npg.nature.com/ reprintsandpermissions/

How to cite this article: Brouwer, I. et al. Direct quantitative detection of Doc2binduced hemifusion in optically trapped membranes. Nat. Commun. 6:8387 doi: 10.1038/ncomms9387 (2015).

cc (i) This work is licensed under a Creative Commons Attribution 4.0 International License. The images or other third party material in this article are included in the article's Creative Commons license, unless indicated otherwise in the credit line; if the material is not included under the Creative Commons license, users will need to obtain permission from the license holder to reproduce the material. To view a copy of this license, visit http://creativecommons.org/licenses/by/4.0/ 